\title{
COLORING SIX-RINGS
}

\author{
BY \\ D. W. HALL AND D. C. LEWIS
}

1. Introduction. In a recent paper by Birkhoff and Lewis, entitled Chromatic polynomials (hereafter denoted $\mathrm{CP})\left({ }^{1}\right)$, there is to be found a rather complete analysis of the four-ring and of the five-ring in the sense that the constrained chromatic polynomials for the part of the map consisting of the ring and one of its sides (say, its exterior) were expressed in terms of free chromatic polynomials. The corresponding analysis for the six-ring was left incomplete because it involves the solution of a very complicated system of fifteen linear equations whose coefficients are polynomials.

In the present paper these fifteen equations, which were only indicated in CP (cf. p. 443), are explicitly written out, and their solution is obtained. The solution gives directly fifteen of the 41 fundamental constrained polynomials for the six-ring. The other 26 constrained polynomials are obtained from these fifteen by substitution in known formulas. The complete set of the 41 constrained polynomials is written out in $\$ 4$.

The mere solution of a system of fifteen linear equations in fifteen unknowns would, of course, be of little mathematical interest. But this particular fifteenth order system, together with the third order system for the fourring and the sixth order system for the five-ring, are special cases of the systems of order $\nu(n)$ for the $n$-ring $\left({ }^{2}\right)$. The solution of this system for the $n$-ring would probably be a major achievement in the future development of the theory of chromatic polynomials. Both the explicit solution for the six-ring and the methods for obtaining this solution will, it is hoped, supply valuable hints for treating the 7- and 8-rings, and eventually the $n$-ring. Moreover, since any constrained polynomial is non-negative for positive integral values of its argument, it is clear that the 41 expressions for the 41 fundamental constrained polynomials for the 6-ring yield numerous inequalities satisfied by free chromatic polynomials for sets of "neighboring" maps (that is, maps differing only slightly from each other in a certain localized portion). To indicate what can be done in this direction, it is enough to refer the reader to $\mathrm{CP}$, p. $420, \S 6$, where the corresponding subject for the 4-ring is given a rather complete discussion. This discussion admits immediate generalization to rings of higher order, when once the fundamental constrained polynomials are expressed in terms of free polynomials.

Presented to the Society, April 26, 1947 ; received by the editors May 27, 1947.

(1) Trans. Amer. Math. Soc. vol. 60 (1946) pp. 355-451. For definitions of the terms "constrained" and "free" polynomials, this paper should be consulted.

(2) For the definition of $\nu(n)$, cf. CP pp. 432-433. An explicit algebraic expression for $\nu(n)$ has not been discovered. It is of interest to observe that $\nu(4)=3, \nu(5)=6, \nu(6)=15, \nu(7)=36$. 
2. The fifteen equations and an outline of their solution. In $C P, p .443$, are given directions for setting up the fifteen equations in the fifteen unknowns $B_{1}, B_{2}, B_{3}, B_{4}, B_{5}, B_{6}, C_{1}, C_{2}, C_{3}, D, E_{1}, E_{2}, I_{1}, I_{2}, I_{3}$. Using also the other notation of $\mathrm{CP}$, as well as the new notation,

$$
B=\sum_{i=1}^{6} B_{i}, \quad C=\sum_{i=1}^{3} C_{i}, \quad E=\sum_{i=1}^{2} E_{i}, \quad I=\sum_{i=1}^{3} I_{i},
$$

the fifteen equations are written as follows:

$$
\left(-u^{2}+u+1\right) B_{1}+\left(u^{2}+u\right)\left(-B_{3}-B_{4}-B_{5}+C+I_{1}\right)
$$

$-\left(u^{2}+2 u\right) B_{i}-u\left(B_{i+1}+B_{i-1}\right)+B_{i+3}+\left(u^{2}+3 u+1\right) C_{i}+u\left(C-C_{i}\right)$

$$
-u^{2} D+u E_{i}+2 E+u I_{i}-I_{i+1}-I_{i-1}=Y_{i}
$$

$$
(i=1,2,3,4,5,6)\left({ }^{3}\right) \text {. }
$$

$$
\begin{aligned}
& -u\left(B_{i}+B_{i+3}\right)+(2 u+1) C_{i}+\left(-u^{2}+u+1\right) D+E \\
& +u\left(I_{i+1}+I_{i-1}\right)=X_{i} \quad(i=1,2,3) . \\
& \left(u^{2}+u\right)\left(B_{i}+B_{i+2}+B_{i-2}\right)+u B-u(C+D)+\left(u^{2}+u\right) E_{i} \\
& -2 E+I=\left(u^{2}+u\right) W_{i} \quad(i=1,2) \text {. } \\
& \left(u^{2}+u\right)\left(B_{i}+B_{i+3}\right)+u B+\left(u^{3}+2 u^{2}+u\right) C_{i}-u C-\left(u^{3}+u^{2}+u\right) D \\
& -2 E+\left(u^{2}+u\right) I_{i}+I=\left(u^{2}+u\right) V_{i} \quad(i=1,2,3) .
\end{aligned}
$$

We may also write down five additional equations obtained by advancing the subscripts in equation (1). These five equations will be linearly dependent upon the 15 already written down, as indicated by the identities

$$
M_{1}=M_{2}=M_{3}=M_{4}=M_{5}=M_{6},
$$

where $M_{i}=Z_{i}+Y_{i+1}+Y_{i-1}+X_{i}+W_{i+1}$. These identities are readily established with the help of the third fundamental principle explained in $\$ 1$, chap. I of CP (pp. 362-363), or they may be verified with the help of equations (5. 9), chap. VI of CP, p. 443. By advancing the subscripts of (1) and then adding all 6 of the equations containing the $Z$ 's, we may replace equation (1) by the following more symmetrical equation:

$$
\begin{gathered}
\left(-4 u^{2}-2 u+1\right) B+2\left(4 u^{2}+3 u\right) C-6\left(2 u^{3}-u\right) D+3(4 u+3) E \\
+2\left(3 u^{2}-u-2\right) I=Z
\end{gathered}
$$

(3) Subscripts are taken modulo 6 for $B, F, G, J, Y, Z$; modulo 3 for $C, H, I, K, X, V$, and modulo 2 for $E$ and $W$. 
where $Z=\sum_{i=1}^{6} Z_{i}$.

The solution of these fifteen equations was considerably simplified by means of certain relations, numbered (8), (9), (10), (11), below, some of which were discovered more or less accidentally in the routine process of elimination. It was discovered later that all except (11) can be derived by direct reasoning based on results already obtained for the 4- and 5-ring. This method will be explained in the next section. For the present, the reader can easily verify a posteriori, with the help of equations (1) to (5), the truth of the following:

$$
\begin{aligned}
& \left(u^{2}+3 u+1\right)\left(2 B_{i}+E_{i}\right)=-Y_{i}+Y_{i+2}+Y_{i-2} \\
& +(u+1)\left(V_{i}-V_{i+1}-V_{i-1}\right)+(u+1)^{2} W_{i} \text {. } \\
& \left(u^{2}+3 u+1\right)\left(D+I_{i}\right)=-Z_{i}+Y_{i}-\left(Y_{i+1}+Y_{i-1}\right)+Y_{i+3}-X_{i} \\
& +(u+1)\left(X_{i+1}+X_{i-1}\right)-V_{i}+W_{i} \text {. } \\
& \left(u^{2}+3 u+1\right)\left[(u+1) C_{i}-u B_{i+3}+E_{i+1}\right] \\
& =-Z_{i}+(u+1) Y_{i+3}+u X_{i} . \\
& \left(u^{2}+3 u+1\right)\left(E_{1}-E_{2}\right) \\
& =Y_{1}-Y_{2}+Y_{3}-Y_{4}+Y_{5}-Y_{6}+\left(u^{2}+1\right)\left(W_{1}-W_{2}\right) \text {. }
\end{aligned}
$$

These relations were used as follows in obtaining the final solution of the original fifteen equations: Advancing subscripts in (8), (9), and (10) and then adding, we obtain the symmetrical relations,

$$
\begin{aligned}
& \left(u^{2}+3 u+1\right)(2 B+3 E)=Y-2(u+1) V+3(u+1)^{2} W \\
& \left(u^{2}+3 u+1\right)(6 D+2 I)=-Z+2(2 u+1) X-2 V+3 W \\
& \left(u^{2}+3 u+1\right)[2(u+1) C-u B+3 E]=-Z+(u+1) Y+2 u X
\end{aligned}
$$

where $Y=\sum_{i=1}^{6} Y_{i}, X=\sum_{i=1}^{3} X_{i}, W=\sum_{i=1}^{2} W_{i}, V=\sum_{i=1}^{3} V_{i}$. Upon eliminating $B$ from (8s) and (10s), we obtain

$$
\begin{aligned}
4\left(u^{2}+3 u+1\right)(u+1) C= & -3\left(u^{2}+3 u+1\right)(u+2) E-2 Z+(3 u+2) Y \\
& +4 u X+3 u(u+1)^{2} W-2 u(u+1) V .
\end{aligned}
$$

We now sum (3) and (5) thus obtaining

$$
-u B+(2 u+1) C+3\left(-u^{2}+u+1\right) D+3 E+2 u I=X
$$

and

$$
\begin{gathered}
\left(u^{2}+4 u\right) B+\left(u^{3}+2 u^{2}-2 u\right) C-3\left(u^{3}+u^{2}+u\right) D-6 E \\
+\left(u^{2}+u+3\right) I=\left(u^{2}+u\right) V .
\end{gathered}
$$

Using the values for $B, C$, and $I$, obtained from (8s), (12), and (9s) in terms of $D$ and $E$, we find that (13) and (14) can be reduced to two equations in two 
unknowns, $D$ and $E$. These equations are

$$
\begin{aligned}
3(u+2) & \left(u^{2}+3 u+1\right) E-12(u+1)\left(u^{2}+u-1\right)\left(u^{2}+3 u+1\right) D \\
= & 2\left(2 u^{2}+4 u+1\right) Z-\left(4 u^{2}+5 u+2\right) Y-4\left(3 u^{3}+4 u^{2}-u-1\right) X \\
& +3 u(u+1)(u-3) W+6 u(u+1) V
\end{aligned}
$$

and

$$
\begin{aligned}
3\left(u^{4}\right. & \left.+6 u^{3}+12 u^{2}+12 u+8\right)\left(u^{2}+3 u+1\right) E \\
& +12(u+1)\left(u^{3}+2 u^{2}+2 u+3\right)\left(u^{2}+3 u+1\right) D \\
= & -2\left(2 u^{3}+4 u^{2}+2 u+3\right) Z+\left(3 u^{4}+10 u^{3}+8 u^{2}+4 u\right) Y \\
& +4\left(3 u^{4}+7 u^{3}+8 u^{2}+10 u+3\right) X \\
& +3(u+1)\left(u^{5}+5 u^{4}+12 u^{3}+18 u^{2}+10 u+6\right) W \\
& -2(u+1)\left(3 u^{4}+12 u^{3}+18 u^{2}+12 u+6\right) V .
\end{aligned}
$$

Equations (15) and (16), upon being solved for $D$, yield

$$
\begin{aligned}
6\left(u^{2}\right. & +3 u+1)\left(u^{3}+4 u^{2}+3 u-1\right) D=-\left(2 u^{2}+8 u+7\right) Z \\
& +2\left(u^{2}+4 u+2\right) Y+2\left(3 u^{3}+10 u^{2}+4 u-1\right) X \\
& +3\left(2 u^{2}+8 u+3\right) W-6\left(u^{2}+3 u+1\right) V .
\end{aligned}
$$

This is one of our original unknowns. Equations (15) and (16) can also be solved for $E=E_{1}+E_{2}$ and then $E_{1}$ can be obtained with the help of (11). Thus

$$
\begin{aligned}
6\left(u^{2}\right. & +3 u+1)\left(u^{3}+4 u^{2}+3 u-1\right) E_{1}=2(2 u+3) Z \\
& +2\left(3 u^{3}+6 u^{2}+u-3\right)\left(Y_{1}+Y_{3}+Y_{5}\right) \\
& -4 u(3 u+4)\left(Y_{2}+Y_{4}+Y_{6}\right)+6\left(u^{5}+4 u^{4}+6 u^{3}+3 u^{2}-u-2\right) W_{1} \\
& +6\left(2 u^{3}-4 u-1\right) W_{2}-4 u X-6(u+1)\left(u^{3}+2 u^{2}-u-1\right) V
\end{aligned}
$$

$E_{2}$ can be obtained by advancing subscripts. We can now find the $B$ 's from (8), the $I$ 's from (9), and the $C$ 's from (10). Thus we have

$$
\begin{aligned}
& 6\left(u^{3}\right.\left.+4 u^{2}+3 u-1\right)\left(u^{2}+3 u+1\right) B_{i}=-(2 u+3) Z+2 u(3 u+4) Y \\
&+2 u X-3\left(2 u^{3}-4 u-1\right) W-6 u(u+1)(u+2) V \\
&-6\left(u^{3}+4 u^{2}+3 u-1\right) Y_{i}+6 u\left(u^{3}+4 u^{2}+3 u-1\right) W_{i} \\
&+6(u+1)\left(u^{3}+4 u^{2}+3 u-1\right) V_{i} . \\
& 6(u+1)\left(u^{2}+3 u+1\right)\left(u^{3}+4 u^{2}+3 u-1\right) C_{i} \\
&=-\left(2 u^{2}+7 u+6\right) Z+2 u\left(3 u^{2}+10 u+8\right) Y+2 u(u+2) X \\
& \quad-3\left(2 u^{4}+6 u^{3}+4 u^{2}-3 u-4\right) W-6(u+1)^{2} V \\
& \quad+6\left(u^{3}+4 u^{2}+3 u-1\right)\left[-Z_{i}-Y_{i+1}-Y_{i-1}+u X_{i}+W_{i}+\left(u^{2}+u\right) V_{i}\right]
\end{aligned}
$$




$$
\begin{aligned}
6\left(u^{2}+3 u+1\right)\left(u^{3}+4 u^{2}+3 u-1\right) I_{i}=\left(2 u^{2}+8 u+7\right) Z \\
\quad-2\left(u^{2}+4 u+2\right) Y+2\left(3 u^{4}+12 u^{3}+11 u^{2}+2 u-2\right) X \\
\quad-3\left(2 u^{2}+8 u+3\right) W+6\left(u^{2}+3 u+1\right) V+6\left(u^{3}+4 u^{2}+3 u-1\right) \\
\quad \cdot\left[-Z_{i}+Y_{i}-Y_{i+1}-Y_{i-1}+Y_{i+3}-(u+2) X_{i}-V_{i}+W_{i}\right] .
\end{aligned}
$$

3. Utilization of results on the 4- and 5-ring to aid in the 6-ring. It is important for building up an inductive theory leading eventually to the solution for the $n$-ring to understand the manner in which information for a ring of lower order can be applied to a ring of higher order. To illustrate the process, we proceed to derive equation (8) from known results on the 4-ring.

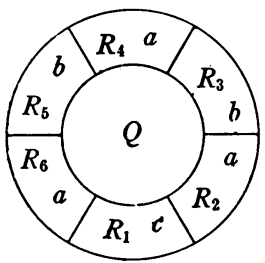

FIG. 1, $B_{1}$

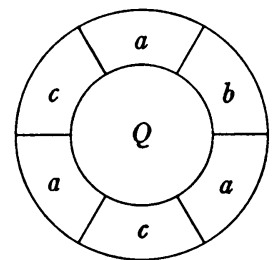

FIG. 2, $B_{3}$

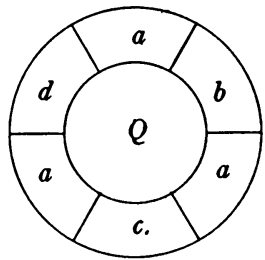

FIG. 3, $E_{1}$

In order to derive (8), we need a relation involving $B_{1}$ and $E_{1}$. These schemes in accordance with the table on p. 441 of $\mathrm{CP}$ may be colored exactly

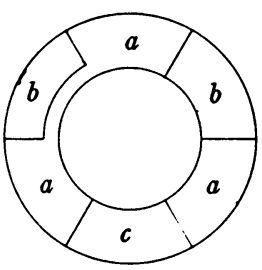

FIG. 4

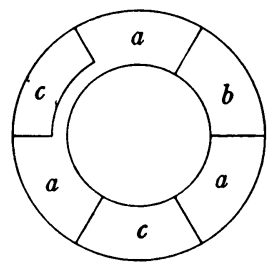

FIG. 5

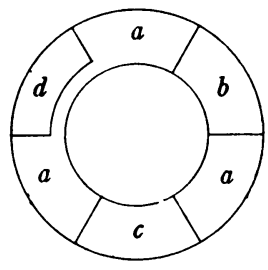

FIG. 6

the same as far as regions $R_{1}, R_{2}, R_{3}, R_{4}$, and $R_{6}$ are concerned, which may be assigned to colors $c, a, b, a$, and $a$, respectively, but region $R_{5}$ in $B_{1}$ is colored $b$, while region $R_{5}$ in $E_{1}$ is colored $d$. Cf. figs. 1 and 3. The only other scheme in which regions $R_{1}, R_{2}, R_{3}, R_{4}$ and $R_{6}$ can be colored in exactly the same way is the scheme $B_{3}$ illustrated in Fig. 2, where the region $R_{5}$ is colored $c$. Since regions $R_{4}$ and $R_{6}$ in all three schemes are colored alike, they may be connected by a corridor as illustrated in Figs. 4, 5, and 6 . We thus obtain a 4-ring consisting of $R_{1}, R_{2}, R_{3}$, and the region $\bar{R}$ consisting of the union of $R_{4}, R_{6}$ and the corridor. We identify this region $\bar{R}$ with the region $R_{4}$ mentioned in the discussion of the 4-ring beginning on p. 415 of CP. It is thus clear, referring to Fig. 24 of CP, p. 416, that the scheme for the four-ring is the one de- 
noted by $A_{3}$ on page 416 and a formula for which is given near the bottom of this same page, to the effect that

$$
\left(\lambda^{2}-3 \lambda+1\right) A_{3}=K_{1}-(\lambda-3) L_{1}+(\lambda-3)(\lambda-1) L_{2} .
$$

Here, of course, $A, K$, and $L$ have entirely different meanings from the $A, K$, and $L$ of the next section. Rewriting (22) in terms of $u=\lambda-3$, we find that

$$
\left(u^{2}+3 u+1\right) A_{3}=K_{1}-u L_{1}+u(u+2) L_{2} .
$$

Since it is obvious from an inspection of Fig. 4; Fig. 5, and Fig. 6 that $B_{1}+B_{3}+E_{1}=A_{3}$, we find at once from (23) that

$$
\left(u^{2}+3 u+1\right)\left(B_{1}+B_{3}+E_{1}\right)=K_{1}-u L_{1}+u(u+2) L_{2} .
$$

It is now necessary to find $K_{1}, L_{1}$, and $L_{2}$ in terms of $Z_{i}, Y_{i}, X_{i}$, and so on. $K_{1}$ is formed by erasing the boundary between $R_{1}$ and $Q_{1}$ after the corridor has been introduced (cf. definition of $K_{1}$, p. 416, of CP). The map $K_{1}$ is illus-

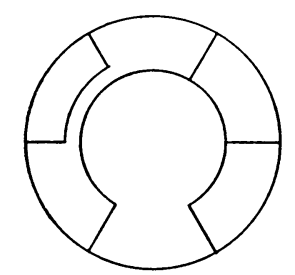

FIG. 7

trated in Fig. 7, and it is readily verified from the third fundamental principle of pp. 362 and 363 of $\mathrm{CP}$ and the definitions of $V_{i}, Y_{i}$, and $W_{i}$ on p. 443(4) of $\mathrm{CP}$ that

$$
K_{1}=-V_{2}+Y_{5}+W_{1}
$$

Similarly we can show even more easily that

$$
L_{1}=V_{2} \text { and } L_{2}=W_{1} .
$$

Hence using (25) and (26) in (24), we see that we have proved that

$$
\left(u^{2}+3 u+1\right)\left(B_{1}+B_{3}+E_{1}\right)=Y_{5}+(u+1)^{2} W_{1}-(u+1) V_{2} \text {. }
$$

Advancing subscripts by 2 units and multiplying by -1 , we have (on remembering that $\left.E_{5}=E_{3}=E_{1}, W_{5}=W_{3}=W_{1}, V_{4}=V_{1}, V_{5}=V_{2}, V_{6}=V_{3}\right)$

(4) Notice misprint on third line of p. 443 of CP where $Y_{1}$ should be replaced by $Y_{i}$. Other misprints and errors in CP are the following: 1. The definition of a proper ring on p. 361 is incomplete at least insofar as 2- and 3-rings are concerned. It should be specified that every proper ring should contain at least one region completely on each side. Thus, the three regions meeting at a (triple) vertex do not form a proper ring. 2 . On p. 425 , line $2, A_{k}^{*}(b c d)$ should be replaced by $A_{k}^{*}(a c d)$. 3. On p. 433 , line 27 , “ $\S 2$ and 5 " should be replaced by “ $\S 5$ and 8. ." 


$$
\left(u^{2}+3 u+1\right)\left(-B_{3}-B_{5}-E_{1}\right)=-Y_{1}-(u+1)^{2} W_{1}+(u+1) V_{1} .
$$

Repeating the same operation gives

$$
\left(u^{2}+3 u+1\right)\left(B_{5}+B_{1}+E_{1}\right)=+Y_{3}+(u+1)^{2} W_{1}-(u+1) V_{3} .
$$

Adding (27), (28) and (29) yields (8) with $i=1$. The other relations can be found by advancing subscripts.

In the same general way equations (9) and (10) were derived. The only difference was that, instead of merging two like colored regions with the help of an "open" corridor, two unlike colored regions were brought into contact with the help of a "closed" corridor. In the latter cases, we were therefore led to the formulas given for the 5-ring instead of those for the 4-ring referred to above.

4. Expressions for the constrained polynomials. Having solved for the $B$ 's, $C$ 's, $D, E$ 's, and $I$ 's, as indicated in $\$ 2$, we are now in a position to write down the other constrained polynomials (namely, $A$, the $F$ 's, $G$ 's, $H$ 's, $J$ 's, $K$ 's, and $L$ ) in terms of the $Z$ 's, $Y$ 's, and so on. This is done with the help of formulas (5. 8), p. 442 of CP. The results seem somewhat simpler when expressed in terms of $x=u+1=\lambda-2$. Putting $f_{1}=u^{2}+3 u+1=x^{2}+x-1$ and $f_{2}=u^{3}+4 u^{2}+3 u-1=x^{3}+x^{2}-2 x-1$, our results are tabulated as follows:

$$
\begin{aligned}
6 f_{1} f_{2} A= & (2 x+1) Z-2\left(3 x^{2}-2 x-1\right) Y-2(x-1) X \\
& +3\left(2 x^{3}-6 x^{2}+2 x+1\right) W+6\left(x^{3}-x\right) V \\
6 f_{1} f_{2} B_{1}= & -(2 x+1) Z+2\left(3 x^{2}-2 x-1\right) Y+2(x-1) X \\
& -3\left(2 x^{3}-6 x^{2}+2 x+1\right) W-6\left(x^{3}-x\right) V \\
& +6 f_{2}\left(-Y_{1}+(x-1) W_{1}+x V_{1}\right) . \\
6 x f_{1} f_{2} C_{1}= & -\left(2 x^{2}+3 x+1\right) Z+2\left(x^{2}-1\right)(3 x+1) Y+2\left(x^{2}-1\right) X \\
& -3\left(2 x^{4}-2 x^{3}-2 x^{2}-x-1\right) W-6 x^{2} V \\
& +6 f_{2}\left[-Z_{1}-Y_{2}-Y_{6}+(x-1) X_{1}+W_{1}+\left(x^{2}-x\right) V_{1}\right] . \\
6 f_{1} f_{2} D= & -\left(2 x^{2}+4 x+1\right) Z+2\left(x^{2}+2 x-1\right) Y+2\left(3 x^{3}+x^{2}-7 x+2\right) X \\
& +3\left(2 x^{2}+4 x-3\right) W-6 f_{1} V . \\
6 f_{1} f_{2} E_{1}= & 2(2 x+1) Z-4\left(3 x^{2}-2 x-1\right) Y-4(x-1) X \\
& -6\left(8 x^{2}-6 x-3\right) W-6 x\left(x^{3}-x^{2}-2 x+1\right) V \\
& +6 f_{2}\left[Y_{1}+Y_{3}+Y_{5}+\left(x^{2}-2 x+4\right) W_{1}+2 W_{2}\right] . \\
& -3\left(4 x^{3}-2 x^{2}-x+1\right) W+6 x^{2} V \\
& +6 f_{2}\left[x^{2} Y_{1}-(x-1)\left(Y_{2}+Y_{6}\right)+x\left(Y_{3}+Y_{5}\right)\right. \\
& \left.+(x-1)^{2} X_{1}+(2 x-1) W_{1}-\left(x^{2}-x\right) V_{1}-(x-1) Z_{1}\right] .
\end{aligned}
$$




$$
\begin{aligned}
& 6 x f_{1} f_{2} G_{1}=-\left(2 x^{3}-5 x-2\right) Z+2\left(3 x^{4}-2 x^{3}-6 x^{2}+2 x+2\right) Y \\
& -2\left(3 x^{4}+2 x^{3}-6 x^{2}-2 x+1\right) X+3\left(4 x^{4}-4 x^{3}-2 x^{2}-x\right) W \\
& -6 x^{3}\left(x^{2}-2\right) V+6 f_{2}\left[x Z_{2}-(x-1)\left(Z_{3}+Z_{4}\right)\right. \\
& -(2 x-1)\left(Y_{2}+Y_{5}\right)+(x-1)^{2}\left(Y_{3}+Y_{4}\right) \\
& \left.+x\left(Y_{1}+Y_{3}\right)+(3 x-1) X_{2}-x W_{2}+x^{2} V_{2}\right] \text {. } \\
& 6 x f_{1} f_{2} H_{1}=\left(2 x^{4}+2 x^{3}+2 x+1\right) Z+2\left(5 x^{4}-x^{3}-9 x^{2}-x+1\right) Y \\
& +2\left(2 x^{4}+2 x^{3}-6 x^{2}-x+1\right) X \\
& -3\left(2 x^{5}-2 x^{4}+4 x^{3}-2 x^{2}+1\right) W+6 x^{4} V \\
& +6 f_{2}\left[-(2 x-1) Z_{1}-x\left(Y_{3}+Y_{5}\right)-(3 x-1)\left(Y_{2}+Y_{6}\right)\right. \\
& \left.-(3 x-1) X_{1}+x^{2}(x-2) V_{1}+(2 x-1) W_{1}\right] \text {. } \\
& 6 f_{1} f_{2} I_{1}=\left(2 x^{2}+4 x+1\right) Z-2\left(x^{2}+2 x-1\right) Y+2\left(3 x^{4}-7 x^{2}+4 x-2\right) X \\
& -3\left(2 x^{2}+4 x-3\right) W+6 f_{1} V+6 f_{2}\left[-Z_{1}+Y_{1}-Y_{2}+Y_{4}\right. \\
& \left.-Y_{6}-(x+1) X_{1}-V_{1}+W_{1}\right] \text {. } \\
& 6 x f_{1} f_{2} J_{1}=(x-2)\left\{\left(2 x^{4}+4 x^{3}+3 x^{2}+3 x+1\right) Z+2\left(2 x^{4}-2 x^{3}-6 x^{2}+1\right) Y\right. \\
& +2\left(2 x^{4}+x^{3}-6 x^{2}+1\right) X-3\left(6 x^{4}+12 x^{3}-13 x^{2}-5 x+1\right) W \\
& +6 x^{3}(x+1) V+6 f_{2}\left[-(2 x-1) Z_{1}+x^{2} Y_{1}-(3 x-1)\left(Y_{2}+Y_{6}\right)\right. \\
& \left.\left.-(3 x-1) X_{1}-x^{2} V_{1}+(5 x-1) W_{1}+2 x W_{2}\right]\right\} \text {. } \\
& 6 f_{1} f_{2} K_{1}=(x-2)\left\{\left(2 x^{3}+6 x^{2}+5 x+1\right) Z-2\left(x^{3}+3 x^{2}+x-1\right) Y\right. \\
& -2\left(4 x^{3}+3 x^{2}-8 x-1\right) X-3\left(2 x^{3}+6 x^{2}+x-3\right) W \\
& +6 x(x+2) V+6 f_{2}\left[(x+1) X_{1}-Z_{2}-Z_{6}-2 Y_{1}\right. \\
& \left.\left.+Y_{2}+Y_{6}+2 W_{2}+V_{1}\right]\right\} \text {. } \\
& 6 f_{1} f_{2} L=(x-2)(x-3)\left\{\left(x^{3}+7 x^{2}+12 x+4\right) Z-2\left(2 x^{3}+5 x^{2}-1\right) Y\right. \\
& -2(x-1)\left(2 x^{2}+4 x+1\right) X-3\left(x^{3}+7 x^{2}+4 x\right) W \\
& \left.+6\left(x^{3}+2 x^{2}+x\right) V\right\} \text {. }
\end{aligned}
$$

All other constrained polynomials can be obtained from the above by advancing subscripts.

These formulas can, of course, be checked by use of any map containing a hexagon for which the constrained polynomials for the six-ring surrounding the hexagon can be easily found independently of the formulas. The simplest such maps are the so-called elementary maps introduced on p. 432 of CP. By choosing a suitable set of 15 such elementary maps, it is possible to obtain a complete geometric check. Each of the formulas listed above has been checked by means of at least two such elementary maps.

UNIVERSITY OF MARYLAND, College Park, Md. 